\title{
Decentralized Power Management of a Hybrid Microgrid Consisting of Solar Panel and Storage Device
}

\author{
Lida Darvish Nasab Bandar ${ }^{1}$, Mahdi Mozaffarilegha ${ }^{2 *}$ \\ ${ }^{1}$ Department of Electrical Engineering, Bandar Abbas Branch, Islamic Azad University, Bandar Abbas, Iran \\ ${ }^{2}$ Department of Electrical Engineering, Kahnooj Branch, Islamic Azad University, Kahnooj, Iran
}

Corresponding Author Email: mozaffarilegha.m@gmail.com

https://doi.org/10.18280/ejee.210403

Received: 8 April 2019

Accepted: 16 July 2019

\section{Keywords:}

energy management system, micro grid, multi agent system, optimization, genetic algorithm

\begin{abstract}
Energy management systems for micro grids (EMS-MG) play an important role for optimized and stable utilizations. Microgrids (MGs) have different types of resources and loads. They can be used as a basic means for achieving improved reliability and serviceability, better power quality, and enhancing the energy efficiency. Decentralized energy management systems for micro grids (DEMS-MG) play a significant role in their optimal and stable operation. In this paper, the proposed strategy for EMS has been implemented for operation of multiple sample microgrids with renewable energy resources alongside the compound power of support such as microturbine and batteries for equalizing the mismatch between power and storage of extra energy when required. The proposed strategy is characterized by concurrent determination of responsive load based on multiagent system alongside genetic algorithm for minimizing the costs related to the microgrid operation, reducing the consumed power of different types of generated loads, and optimal production of each of the generation resources and the storage device present in the microgrid.
\end{abstract}

\section{INTRODUCTION}

In recent years, due to the progressive need to electricity, application of renewable energy resources such as wind, solar, and other sources has increased in order to enhance the reliability, improve the power quality, and ameliorate the flexibility and to fulfill lower electricity price and less environmental impacts. There are distributed energy resources (DERs) located in the proximity of loads which are categorized into distributed generation (DG) units and distributed storage (DS) units. Systems composed of DERs which can operate in two ways including islanded state and connected to the entire electricity grid are called microgrid (MG). A microgrid is applied as a set of electric loads of nonresponsive loads (NRL), responsive load demands (RLD), and normal loads as well as in the form of DER units equipped with a distribution feeder. The energy management system in the microgrid attempts to maximize the use of renewable resources instead of fossil resources. The reason is that the former does not use fuel and does not contaminate the environment either. Furthermore, it should ensure the reliability of the system given the energy planning. The issue of energy management in microgrids and use of multiagent systems have been frequently proposed for solving it and making decision for energy management.

There are many economical advantages for using the MGs including:

1) Reducing the transmission, distribution and energy loss costs

2) Improving the energy efficiency

3) Low investment costs for private sector and therefore, reduced investment risk Energy management system in $\mathrm{MG}$ tries to maximize the usage of the renewable energies instead of fossil fuels since they do not consume fuel and their environmental pollution is minimal.

Moreover, it must guarantee the reliability of the system with respect to the energy planning. The MG's energy management systems and multi agent systems have been addressed in the literature in order to solve the decision making for energy management.

The differences of centralized and decentralized multiagent systems have been discussed [1]. Then, the advantages of decentralized multiagent systems have been expressed, and concerning the characteristics of microgrid consisting of components and elements with various features and objectives, decentralized multiagent systems method has been chosen and presented for operation and control of the microgrid. In addition, the presence of two operational states including connected to network and islanded operation causes complication of microgrid management and control. This again highlights the importance of using decentralized control and multiagent systems. In the connected to the main grid state, the agents should control generation, load, and storage from a power distribution perspective. The control structure of DERs has been discussed based on multiagent systems [2]. In this way, they introduced three levels of agents in the control structure. The lower levels were considered as local agents (LAs), which directly dealt with controlling DER units and integrated loads. Local agents consider a confined environment and only control special elements. Two layers of DER control strategies have been considered [3]. The primary control focuses on local control, which means maximizing the independence of local agents in order to ensure the security and reliability of the system even when the system communications have found problem. The secondary control focuses on regional or even overall optimization of the system. 
This means that high-level agents are responsible for coordinating the behaviors of local agents considering the general benefits of the system. Therefore, in primary control, individual and autonomous operations can be considered as emergency operations, while in secondary control they cause the system to remain complete and continue its activity. In this reference, microgrid control strategies have been presented in islanded and connected to grid operations. In the latter, the microgrid frequency is adjusted according to the main grid, and the distributed generation units can keep the output active power constant and only control the reactive power. In the secondary control, voltage stability is of priority. The reactive power demand required among DER units is shared so that the voltage would be maintained within an acceptable safe range. The storage local agents decide on either absorbing or releasing energy given their charge capacity and the current load demand. Renewable energy resources are an important component of a microgrid. Storage systems such as capacitors, batteries, flywheels, etc. have also great applications in a microgrid, especially when renewable resources have been used in the microgrid. The reason is that they can compensate for the uncertainty of these resources through energy storage and timely delivery of energy to the grid loads. Some solutions and points have been mentioned about coordination between renewable units and storage systems $[4,5]$.

In this paper, to analyze the problem, several scenarios have been followed for decentralized power management of a hybrid microgrid. In the approach introduced for decentralized energy management of a hybrid microgrid, command is made for each of the resources and loads based on the measured data instead of receiving the power commands from central control unit [6].

\section{THE STRUCTURE OF THE TESTED MICROGRID}

In this study, the microgrid consists of a microturbine and batteries as the support system and energy storage system respectively alongside different types of loads. The schema of the studied microgrid is shown in Figure 1. Furthermore, this microgrid has been connected to the global electricity grid. Every component of the studied system has been modeled separately based on the relevant characteristics and constraints.

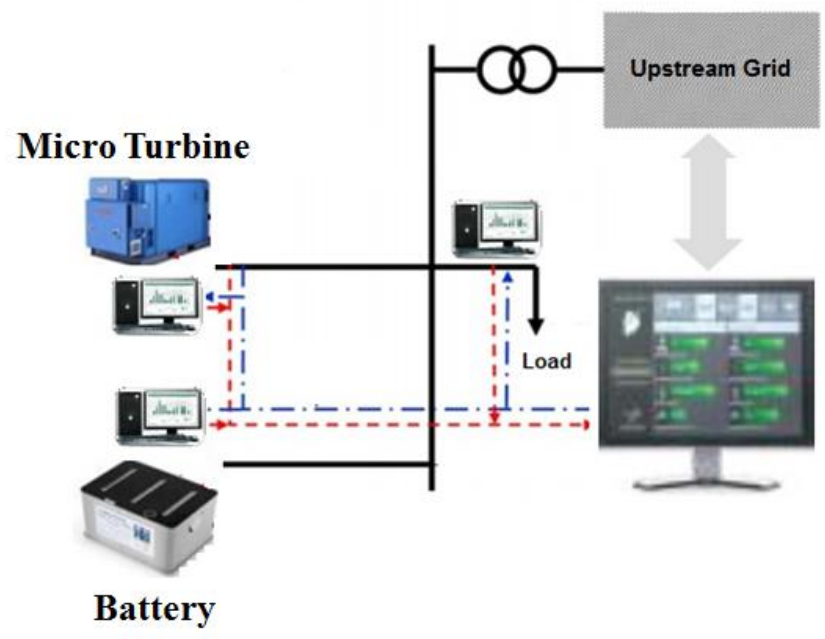

Figure 1. The general schema of the studied microgrid

\section{PROBLEM FORMULATION}

\subsection{Objective function}

For mathematical implementation of the model of interest, the objective function is expressed for maximizing the profit of solar panel and storage device units and for meeting the constraints.

$$
F=\sum_{t=1}^{24}\left[\begin{array}{c}
\sum_{h=1}^{a} P_{t, k}^{M T} \times \pi_{t, k}^{M T}+ \\
\sum_{m=1}^{b} P_{t, m}^{G R I D-} \times \pi_{t, m}^{G R I D-}+\sum_{n=1}^{c} P_{t, n}^{G R I D+} \times \pi_{t, n}^{G R I D+}+ \\
\sum_{l=1}^{d} P_{t, l}^{B T-} \times \pi_{t, l}^{B T-}+\sum_{p=1}^{e} P_{t, p}^{B T+} \times \pi_{t, p}^{B T+}+ \\
\sum_{o=1}^{f} P_{t, o}^{U P} \times \pi_{t, o}^{U P}
\end{array}\right]
$$

In this relation (1), parameter a represents the number of photovoltaic units, $b$ shows the number of times power has been sold to the global grid, c shows the number of times power has been purchased from the global grid, $d$ denotes the number of times the energy storage system has been discharged, e refers to the number of times the energy storage system has been charged, and f shows the frequency of no load responsivity.

\subsection{Operational constraints}

$$
\mathrm{P}_{\mathrm{t}}^{\mathrm{MT}} \leq \bar{P}_{\mathrm{t}}^{\mathrm{MT}}
$$

Relation 2 indicates that $\bar{P}_{\mathrm{t}}^{\mathrm{MT}}$ is the maximum power generated by a MT unit within the time range of $t$.

$$
\begin{aligned}
& \mathrm{P}_{\mathrm{t}}^{\mathrm{BT}-} \leq \bar{P}_{\mathrm{t}}^{\mathrm{BT}-} \\
& \mathrm{P}_{\mathrm{t}}^{\mathrm{BT}+} \leq \bar{P}_{\mathrm{t}}^{\mathrm{BT}+}
\end{aligned}
$$

Relations (3) and (4) indicate that BT in discharging state should be lower than the maximum discharging power, while in the charging state, it should be lower than the maximum charging power. Note that the charge status of SOC battery during the time of $t$ is equal to the energy stored within the time of $\mathrm{t}$ divided by the total stored energy, which has been shown in Relation (5).

$$
\begin{gathered}
S O C_{t}=\frac{\mathrm{E}_{\mathrm{t}}^{\mathrm{BT}}}{\mathrm{E}_{e q}^{\mathrm{BT}}} \\
P_{\mathrm{t}}^{\mathrm{TCP}}=\mathrm{P}_{\mathrm{t}}^{\mathrm{GRID}-}+\mathrm{P}_{\mathrm{t}}^{\mathrm{BT}-}
\end{gathered}
$$

Relation (6) reveals that is the total consumed power.

$$
P_{\mathrm{t}}^{\mathrm{TGP}}=\mathrm{P}_{\mathrm{t}}^{\mathrm{MT}}+\mathrm{P}_{\mathrm{t}}^{\mathrm{GRID}+}+\mathrm{P}_{\mathrm{t}}^{\mathrm{BT}+}
$$

Relation (7) indicates that is the total generated power.

$$
\mathrm{P}_{\mathrm{t}}^{\mathrm{GRID}+}+\mathrm{P}_{\mathrm{t}}^{\mathrm{BT}+}+\mathrm{P}_{\mathrm{t}}^{\mathrm{MT}}=\mathrm{P}_{\mathrm{t}}^{\mathrm{GRID}-}+\mathrm{P}_{\mathrm{t}}^{\mathrm{BT}-}
$$


In order to constrain the power interchanges between the microgrid and upstream grid and for better usage of the resources available in the microgrid, Relation (8) has been considered, where $\mathrm{P}$ interchange is the interchanged power.

\section{DECENTRALIZED POWER MANAGEMENT STRATEGY}

Agent is a computer system that is capable of performing independent and automatic operations by the user or owner. The main point regarding the agents is their independence; therefore, it can be said that an agent is a computer program that can perform independent operations in some environments [7, 8]. Multi agent system is a novel method for solving and implementing computer software projects. Although these systems are rather new, using design methods based on agents has been one of the most successful solutions and resulted in the distributed solving systems which is considered as one of the best available systems [9, 11, 12]. There are two important problems in designing MAS; first is the designing of the agent and second is an environmental design for the performance and communication between the agents. In agent design, the steps for creating an agent that is able to perform independent task and duties is considered such that it can successfully perform the desired tasks. For environmental design, the major point is that how could these agents be designed such that they can communicate with each other. The meaning of communication is cooperation, coordination and negotiation between the agents [10, 13].

The MAS technology in MGs has been vastly deployed and improved. The performance of these systems in troubleshooting and fault detection, maintaining stability, grid repair, grid control, market simulation and automization is a proof for this claim [14]. One of the required factors for the MGs is that flexibility and expandability of the solutions. Given that the MAS are flexible, expandable and fault proof, they are a suitable choice in MGs.

In order to address the active power management problem, this paper proposes a Multi-Agent System [15, 16] for microgrid, according to the concept shown in Figure 2.

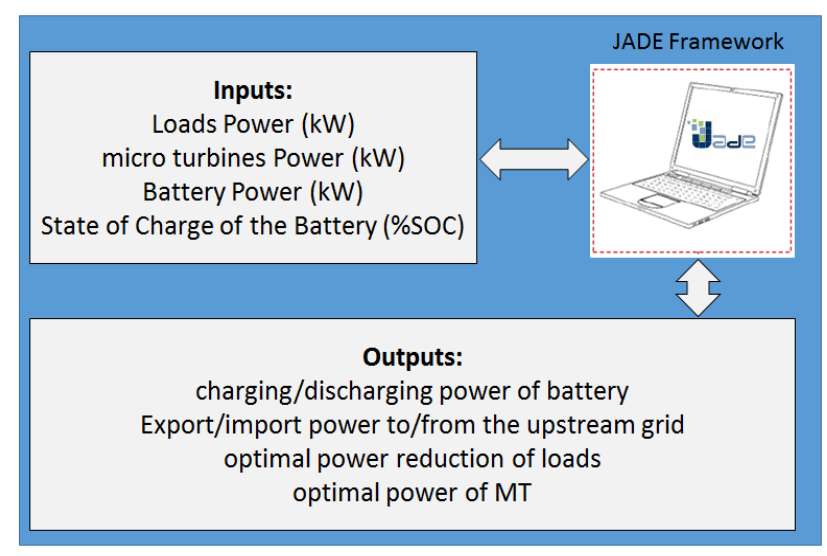

Figure 2. Flowchart of the proposed EMS strategy

In this problem, the goal is to find the power values of distributed generation units including solar panel and load storage device [17, 18]. Furthermore, in the introduced hybrid microgrid decentralized energy management, the commands are issued for each of the resources and loads based on the measured data instead of receiving power commands from a central control unit $[19,20]$. The proposed strategy leads to minimization of the objective function through optimizing the problem variables. Table 1 indicates the stages of the decentralized power management strategy.

Table 1. Decentralized power management strategy stages

1. Initialization
2. If $T G P>T C P$
i. If $T G P-T C P<\bar{P}_{\mathrm{t}}^{\text {ES+ }}$
ESS charging and storage of new SOC
a. Do we have still extra generation?
Responsivity to load and, if possible, selling to the upstream grid
b. Otherwise, termination.
ii. load responsivity and the possible selling to the upstream grid
3. If $T G P<T C P$
i. Is power deficit greater than the minimum MT power?
a. It the power deficit greater than the maximum MT?
Maximum MT capacity and, if possible, purchasing from the grid
b. Otherwise, turning MG on as much as the required power.
ii. Is the power deficit less than the maximum charging power?
a. Discharging stored ESS of new SOC
b. Otherwise, if the conditions for purchasing from grid were not
met, shift to another time

The upstream grid is controlled by the microgrid Agent (MG Agent) and according to Figure 3, the proposed method must be performed according to the decentralized power management strategy.

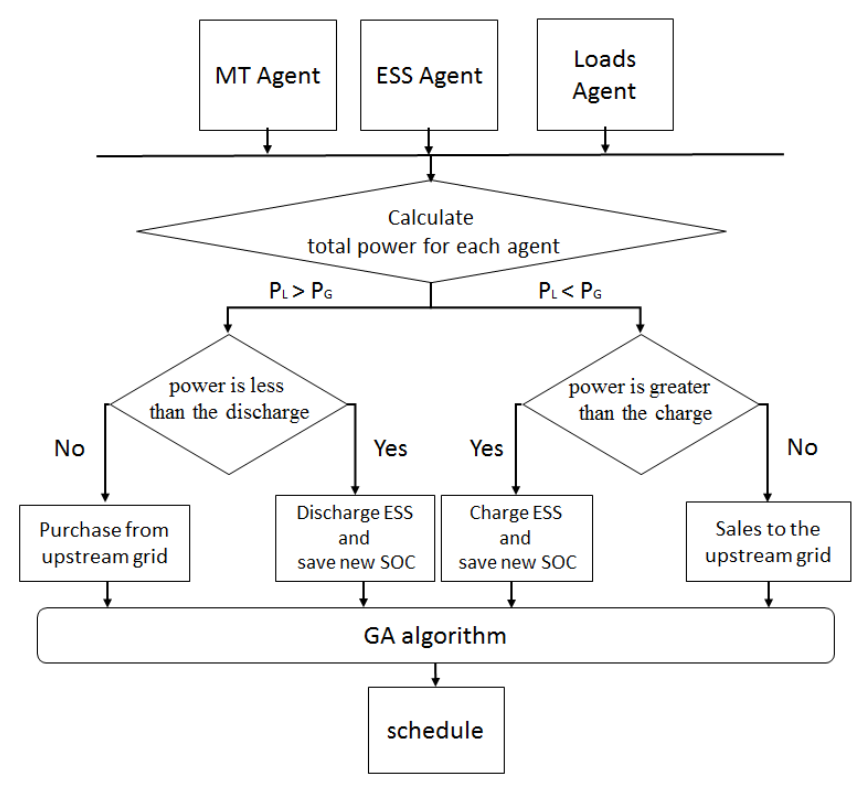

Figure 3. Flowchart of the proposed EMS strategy

The EMS-MG should meet the following requirements. In the grid-connected operation mode, the EMS should be able to dynamically determine the power exchanged between the micro grid and the upstream grid in the islanded operation mode, the EMS should be realized to ensure the balance of power and the stability of the frequency.

Ensure the safety of operation of micro grid in gridconnected mode and islanded mode. Based on the above requirements, the EMS-MG can be divided into three levels participants: level 1) the system control Agent; level 2) the MG Agent; and level 3) the Local Agent, as shown in Figure 4. 


\section{RESULTS AND DISCUSSION}

In this section, the results obtained from evaluation of the proposed strategy on the studied microgrid have been presented. Then, some of the outputs are also analyzed. Figure. 5 demonstrates the power generation diagram within a 24-hour operation of the sample microgrid. As can be observed, only in three ranges, the microgrid sold power to the global electricity grid. Figure 6 displays the power consumption diagram within a 24-hour operation of the sample microgrid. As can be seen, only within three times ranges when the load demand value has been less than the magnitude generated by the microgrid, first the proposed strategy charged ES, and then responded to the magnitude of the power shifted from the previous stage. Within the power deficit intervals, this has occurred because of the lower deficit value compared to the minimum microturbine value. Figure 7 illustrates the SOC value along the daily operation of the system. As can be observed, SOC in more than $55 \%$ of the daily operation has been above $50 \%$, suggesting very suitable performance of the proposed strategy during the day.

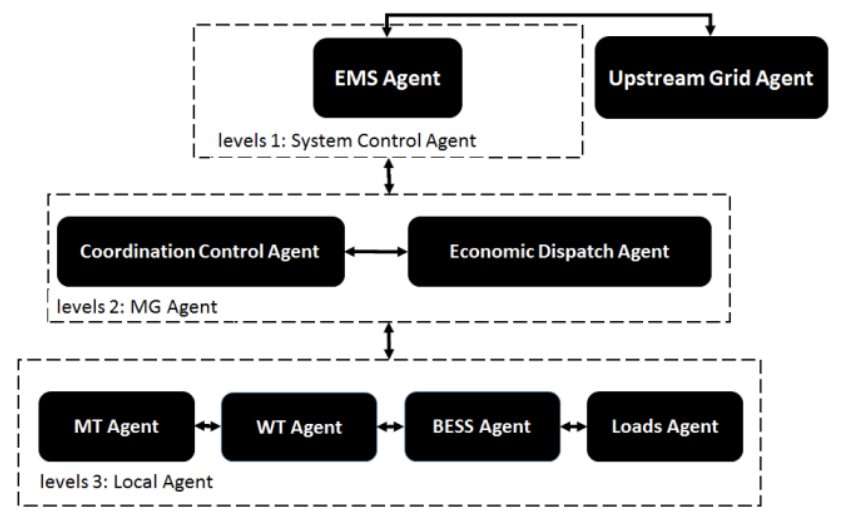

Figure 4. Structure of the proposed EMS-MG

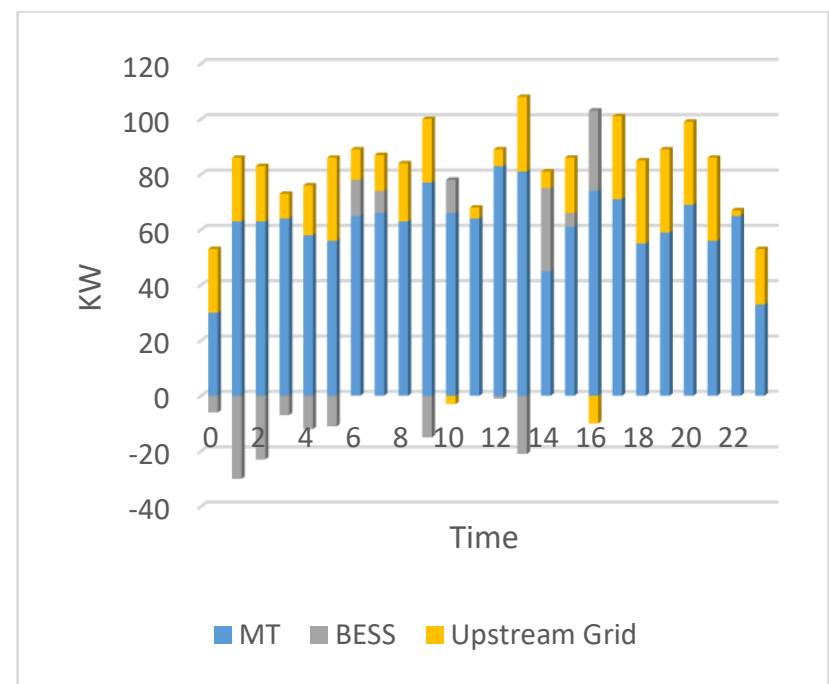

Figure 5. The paragraph of power generation within the 24hour operation of the system

\section{CONCLUSION}

Energy management systems for micro grids (EMS-MG) play an important role for optimized and stable utilizations.
Microgrids (MGs) have different types of resources and loads. They can be used as a basic means for achieving improved reliability and serviceability, better power quality, and enhancing the energy efficiency.

In this paper, the proposed strategy has been presented with a new objective function for decentralized power management of a hybrid microgrid consistent of solar panels and storage device. By adding some constraints to the optimization problem, some considerations were adopted for fulfilling the objectives of interest. The potential of the proposed method has been confirmed according to the obtained results. Indeed, these results suggest that the strategy proposed in this paper has a good potential in reducing costs and meeting the needs of consumers.

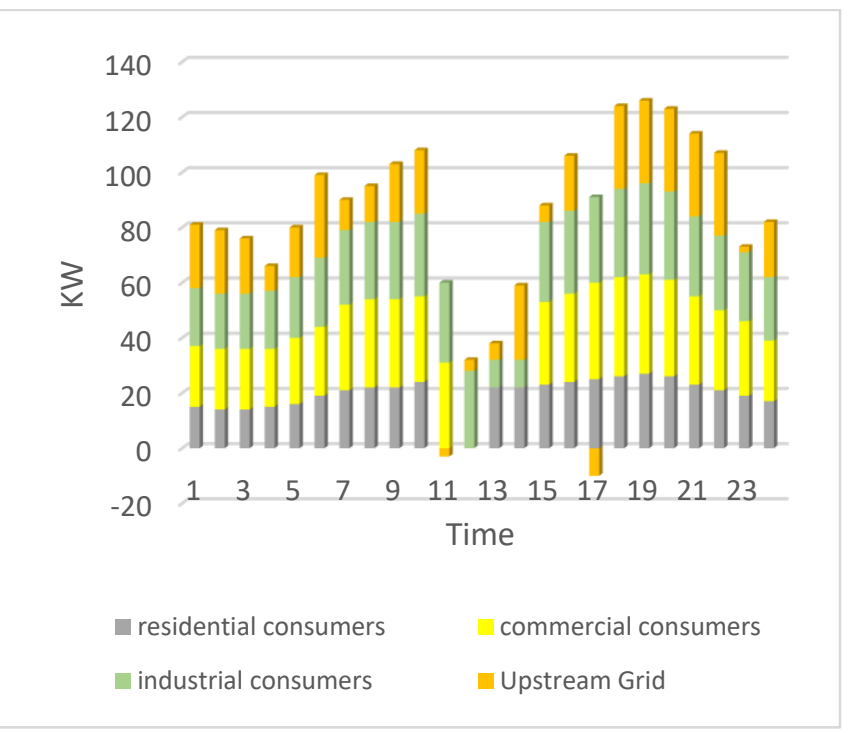

Figure 6. The paragraph of power consumption within the 24-hour operation of the system

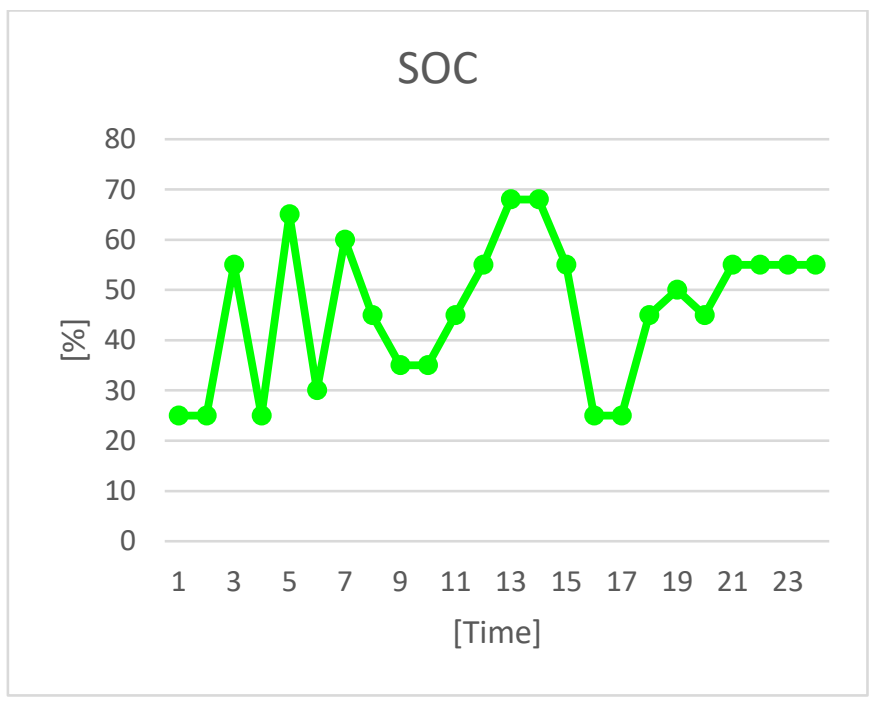

Figure 7. SOC along the daily operation of the system

\section{REFERENCES}

[1] Colson, C.M., Nehrir, M.H. (2011). Algorithms for distributed decision-making for multi-agent microgrid power management. Power and Energy Society General Meeting, 2011 IEEE, pp. 1-8. 
http://dx.doi.org/10.1109/PES.2011.6039764

[2] Zheng, W.D., Cai, J.D. (2010). A multi-agent system for distributed energy resources control in microgrid. Conference on Critical Infrastructure (CRIS), 2010 5th International, pp. 1-5.

[3] Colson, C.M., Nehrir, M.H., Gunderson, R.W. (2011). Multi-agent microgrid power management. Preprints of the 18th IFAC World Congress Milano (Italy). http://dx.doi.org/10.3182/20110828-6-IT-1002.01188

[4] Cha, H.J., Choi, J.Y., Won, D.J. (2014). Smart load management in demand response using microgrid EMS. Energy Conference (ENERGYCON), IEEE International, pp. 833-837. http://dx.doi.org/10.1109/ENERGYCON.2014.6850522

[5] Shehata, E.G., Gaber, M.S., Ahmed, K.A., Salama, G.M. (2018). Implementation of an energy management algorithm in DC MGs using multi-agent system. Electrical Energy Systems, 29(4). http://dx.doi.org/10.1002/etep.2790

[6] Diaz, N.L., Luna, A.C., Vasquez, J.C., Guerrero, J.M. (2017). Centralized control architecture for coordination of distributed renewable generation and energy storage in islanded AC microgrids. IEEE Trans. Power Electron. 32(7): $5202-5213$ http://dx.doi.org/10.1109/TPEL.2016.2606653

[7] Marzband, M. Sumper, A., Domínguez-García, J.L., Gumara-Ferret, R. (2015). Experimental validation of a real time energy management system for microgrids in islanded mode using a local day-ahead electricity market and MINLP. Energy Convers, 76: 314-322. http://dx.doi.org/10.1016/j.enconman.2013.07.053

[8] Marzband, M., Azarinejadian, F., Savaghebi, M., Guerrero, J.M. (2014). An optimal energy management system for islanded microgrids based on multiperiod artificial bee colony combined with Markov chain. IEEE, 11(3). http://dx.doi.org/10.1109/JSYST.2015.2422253

[9] Shimomachi, K., Noritake, M., Hara, R., Hoshi, H., Kita, H., Hirose, K. (2014). Development of energy management system for DC microgrid for office building: Day ahead operation scheduling considering weather scenarios. Power Systems Computation Conference (PSCC), pp. $1-6$. http://dx.doi.org/10.1109/PSCC.2014.7038313

[10] Tarique, T.A., Zamee, M.A., Khan, M.I. (2014). A new approach for pattern recognition with Neuro-Genetic system using Microbial Genetic Algorithm. Electrical Engineering and Information \& Communication Technology (ICEEICT), 2014 International Conference pp.

http://dx.doi.org/10.1109/ICEEICT.2014.6919082

[11] Lin, B., Zhou, M., Du, W., Liu, C. (2013). Improved PSO algorithm for microgrid energy optimization dispatch. Power and Energy Engineering Conference (APPEEC), 2013 IEEE PES Asia-Pacific, pp. 1-6.

[12] Dou, C., Jia, X., Bo, Z., Zhao, F., Liu, D. (2011). Optimal management of micro grid based on a modified particle swarm optimization algorithm. Power and Energy Engineering Conference (APPEEC), 2011 Asia-Pacific, pp.

$1-8$. http://dx.doi.org/10.1109/APPEEC.2011.5747690

[13] Farzin, H., Ghorani, R., Fotuhi-Firuzabad, M., MoeiniAghtaie. (2017). A market mechanism to quantify emergency energy transactions value in a multimicrogrid system. IEEE Transactions on Sustainable Energy, 10(1): 426-437. http://dx.doi.org/10.1109/TSTE.2017.2741427

[14] Mozaffarilegha, M., Farjah, E. (2018). Implementation of energy management of a microgrid using HMAS. 2018 Smart Grid Conference (SGC). http://dx.doi.org/10.1109/SGC.2018.8777876

[15] Turky, A.M., Abdullah, S., Sabar, N.R. (2014). A hybrid harmony search algorithm for solving dynamic optimisation problems. Procedia Comput. Sci., 29: 19261936. http://dx.doi.org/10.1016/j.procs.2014.05.177

[16] Legha, M.M., Farjah, E. (2019). IOT based load management of a micro-grid using arduino and HMAS. Iranian Journal of Electrical and Electronic Engineering.

[17] Geem, Z.W., Kim, J.H., Loganathan, G.V. (2001). A new heuristic optimization algorithm: Harmony search. Simulation, $\quad 76(2)$ : 60-68. http://dx.doi.org/10.1177/003754970107600201

[18] Zhang, J., Ai, Q., Jiang, C.W., Wang, X.G., Zheng, Z.H., $\mathrm{Gu}, \mathrm{C} . \mathrm{H}$. (2009). The application of multi agent system in microgrid coordination control. Conference on Sustainable Power Generation and Supply, IEEE, pp. 16. http://dx.doi.org/10.1109/SUPERGEN.2009.5348277

[19] Olivares, D.E., Cañizares, C.A., Kazerani, M. (2014). A centralized energy management system for isolated microgrids. IEEE Transactions on Smart Grid, 5(4): 1864-1875. http://dx.doi.org/10.1109/TSG.2013.2294187

[20] Elsied, M., Oukaour, A., Gualous, H., Youssef, T., Mohammed, M. (2014). An advanced energy management of microgrid system based on genetic algorithm. Industrial Electronic (ISIE), 114: 742-752. https://doi.org/10.1016/j.energy.2016.08.048 